\title{
Large Margin Image Set Representation and Classification
}

\author{
Jim Jing-Yan Wang, Majed Alzahrani and Xin Gao
}

\begin{abstract}
In this paper, we propose a novel image set representation and classification method by maximizing the margin of image sets. The margin of an image set is defined as the difference of the distance to its nearest image set from different classes and the distance to its nearest image set of the same class. By modeling the image sets by using both their image samples and their affine hull models, and maximizing the margins of the images sets, the image set representation parameter learning problem is formulated as an minimization problem, which is further optimized by an expectation-maximization (EM) strategy with accelerated proximal gradient (APG) optimization in an iterative algorithm. To classify a given test image set, we assign it to the class which could provide the largest margin. Experiments on two applications of video-sequence-based face recognition demonstrate that the proposed method significantly outperforms state-of-the-art image set classification methods in terms of both effectiveness and efficiency.
\end{abstract}

\section{INTRODUCTION}

$\mathbf{T}$ RADITIONAL visual information processing and understanding is based on single images. The classification of a visual target, such as a human face, is also conducted based on a single image, where each training or test sample is an individual image [1], [2], [3], [4]. With the rapid development of video technologies, sequences of images is more commonly available than single images. Consequently, the visual target classification task could be improved from image-based to image-set-based. The image set classification problem has been proposed and studied recently [5], [6], [7], [8], [9], [10], [5], [11]. In image set classification, each sample is a set of images instead of one single image, and each class is represented by one or more training samples. The classification problem is to assign a given test sample to one of the known classes. For example, in image-set-based face recognition problem, each sample is a set of facial images with different poses, illuminations, and expressions. Compared to the traditional single image classification, image set classification has the potential of achieving higher accuracy, because image sets usually contain more information than single images. Even if images in the image set are of low quality, we could still

Jim Jing-Yan Wang is with the University at Buffalo, The State University of New York, Buffalo, NY 14203, USA, and Provincial Key Laboratory for Computer Information Processing Technology, Soochow University, Suzhou, 215006, China. (E-mail: jimjywang@ gmail.com.)

Majed Alzahrani and Xin Gao are with the Computer, Electrical and Mathematical Sciences and Engineering Division, King Abdullah University of Science and Technology (KAUST), Thuwal 23955-6900, Saudi Arabia. (Email: \{majed.alzahrani, xin.gao\}@kaust.edu.sa.) Xin Gao is the corresponding author. Correspondence should be addressed to Xin Gao.

This study was supported by a grant from King Abdullah University of Science and Technology (KAUST), Saudi Arabia, and Provincial Key Laboratory for Computer Information Processing Technology, China (Grant No. KJS1324). exploit the temporal relationship and the complementarity between the images to improve the classification accuracy.

Although image set classification has proposed a novel and promising scheme for the visual classification problem, it has also brought challenges to the machine learning and computer vision communities. Traditional single-imagebased representation and classification methods are not suitable for this problem, such as principal component analysis (PCA) [12], support vector machine (SVM) [13], and Knearest neighbors (KNN) [14]. To handle the problem of image set classification, a number of methods have been proposed. In [8], Kim et al. developed the discriminant canonical correlations (DCC) for image set classification, by proposing a linear discriminant function to simultaneously maximize the canonical correlations of within-class sets and minimize the canonical correlations of between-class sets. The classification is done by transforming the image sets using the discriminant function and comparing them by the canonical correlations. In [5], Wang et al. formulated the image set classification problem as the computation of manifold-manifold distance (MMD), i.e., calculating the distance between nonlinear manifolds, each of represented one image set. In [15], Wang et al. presented the manifold discriminant analysis (MDA) for the image set classification problem, by modeling each image set as a manifold and formulating the problem as a classification-oriented multimanifold learning problem. Cevikalp and Triggs later introduced the affine hull-based image set distance (AHISD) for image set based face recognition [16], by representing images as points in a linear or affine feature space and characterizing each image set by a convex geometric region spanned by its feature points. Set dissimilarity was measured by geometric distances between convex models. Hu et al. proposed to represent each image set as both the image samples of the set and their affine hull model [17], [18], and introduced a novel between-set distance called sparse approximated nearest point (SANP) distance for the image set classification. In [19], Wang et al. modeled the image set with its natural second-order statistic, i.e. covariance matrix (COV), and proposed a novel discriminative learning approach to image set classification based on the covariance matrix.

Among all these methods, affine subspace-based methods, including AHISD [16] and SANP [17], [18], have shown their advantage over the other methods. However, all these methods are unsupervised ones, ignoring the class labels of the images sets in the training set. Moreover, most imageset-based classification methods are under the framework of pairwise image set comparison [5], [15], [16], [17], [18]. A similar approach has been successfully adopted on pairwise 
comparison on individual samples by $\mathrm{Mu}$ et al. [20], which exploits abundant discriminative information for each local manifold. In set classification case, a test image set is compared to all the training image set one by one, and then the nearest neighbor rule is utilized to decide which class the test image set belongs to. The disadvantage of this strategy lays in the following two folds:

- When the training image set number is large, this strategy is quite time-consuming.

- When a pair of image sets are compared, all other image sets are ignored, and thus the global structure of the image set dataset is ignored.

To overcome these issues, in this paper, we propose a novel image set representation and classification method. Similarly to SANP [17], [18], we also use the image samples of an image set and their affine hull model to represent the image set. To utilize the class labels of each image set, inspired by large margin framework for feature selection [?], we propose to maximize the margin of each image set. Based on this representation and its corresponding pairwise distance measure, we define two types of nearest neighboring image sets for each image set — the nearest neighbor from the same class (nearest hit) and the nearest neighbor from different classes (nearest miss). The margin of a image set is defined as the difference between its distances to nearest miss and nearest hit, and the representation parameter is learned by maximizing the margins of the image sets. To classify a test image set, we assign it to the class which could achieve the largest margin for it. The contributions of the proposed Large Margin Image Set (LaMa-IS) representation and classification method are of three folds:

1) Using the class labels, we define the margin of the image sets, such that the discriminative ability can be improved in a supervised manner.

2) The global structure of the image sets can also be explored by searching the nearest hit and nearest miss from the entire database for each images set.

3) To classify a test image set, we only need to compare it to every class instead of every training image set, which could reduce the time complexity of the online classification procedure significantly, especially when the number of training image sets are much larger than the number of classes.

The rest of the paper is organized as follows: in Section II, we propose the novel LaMa-IS algorithm; in Section III, the experiment results on several image-set-based face recognition problems are given; and finally in Section IV, we draw conclusions.

\section{Methods}

In this section we will introduce the proposed LaMa-IS method for image set representation and classification.

\section{A. Objective Function}

Suppose we have a database of image sets denoted as $\left\{\left(X_{i}, y_{i}\right)_{i=1}^{N}\right\}$, where $X_{i}$ is the data matrix of the $i$-th image set, and $y_{i} \in\{1, \cdots, C\}$ is its corresponding class label. In the data matrix $X_{i}=\left[\mathbf{x}_{i, 1}, \cdots, \mathbf{x}_{i, N_{i}}\right] \in \mathbb{R}^{D \times N_{i}}$, the $n$ th column, $\mathbf{x}_{i, n} \in \mathbb{R}^{D}$ is the $D$-dimensional visual feature vector of the $n$-th image of the $i$-th image set, and $N_{i}$ is the number of images in $i$-th image set. Note that the feature vector for a image can be the original pixel values or some other visual features extracted from the image, such as local binary pattern (LBP) [21]. To represent an image set, two linear model has been employed to approximate the structure of the image set following [17], [18]:

- Using the images in the image set, we can model the $i$-th image set as an linear combination of the images in the $i$-th set as

$$
\mathbf{x}=\sum_{n=1}^{N_{i}} \mathbf{x}_{i, n} \alpha_{i, n}=X_{i} \boldsymbol{\alpha}_{i}
$$

where $\boldsymbol{\alpha}_{i}=\left[\alpha_{i, 1}, \cdots, \alpha_{i, N_{i}}\right]^{\top} \in \mathbb{R}^{N_{i}}$ is the linear combination coefficient vector.

- We can also use the affine hull model to represent the image set, using the image mean and the orthonormal bases of the $i$-th image, which is represented as

$$
\mathbf{x}=\boldsymbol{\mu}_{i}+U_{i} \mathbf{v}_{i}
$$

where $\boldsymbol{\mu}_{i}=\frac{1}{N_{i}} \sum_{n=1}^{N_{i}} \mathbf{x}_{i, n}$ is the image mean, the columns of $U_{i}$ are the orthonormal bases obtained from the SVD of the centered $X_{i}$, and $\mathbf{v}_{i}$ is the coefficient vector of $U_{i}$.

In this paper, we try to represent the image sets using both of the models mentioned above simultaneously, by solving the parameters $\boldsymbol{\alpha}_{i}$ and $\mathbf{v}_{i}$. The representation error is given by the squared $l_{2}$ norm distance between these two models as

$$
\mathcal{R}_{\mathbf{v}_{i}, \boldsymbol{\alpha}_{i}}=\left\|\left(\boldsymbol{\mu}_{i}+U_{i} \mathbf{v}_{i}\right)-X_{i} \boldsymbol{\alpha}_{i}\right\|_{2}^{2},
$$

where $\boldsymbol{\alpha}_{i}$ and $\mathbf{v}_{i}$ are the parameters for the two models, respectively. To compare a pair of image sets, we only use the second model and compute the squared $l_{2}$ norm distance between them suggested by [17] as

$$
\mathcal{D}_{\mathbf{v}_{i}, \mathbf{v}_{j}}=\left\|\left(\boldsymbol{\mu}_{i}+U_{i} \mathbf{v}_{i}\right)-\left(\boldsymbol{\mu}_{j}+U_{j} \mathbf{v}_{j}\right)\right\|_{2}^{2} .
$$

Given the defined distance function $\mathcal{D}_{\mathbf{v}_{i}, \mathbf{v}_{j}}$, we can find two nearest neighboring sets for each set $X_{i}$, one from the same class as $X_{i}$ (called nearest hit or $\mathcal{H}_{i}$ ) and the other from different classes (called nearest miss or $\mathcal{M}_{i}$ ), defined as

$$
\begin{aligned}
\mathcal{H}_{i} & =\underset{j: y_{j}=y_{i}, j \neq i}{\operatorname{argmin}} \mathcal{D}_{\mathbf{v}_{i}, \mathbf{v}_{j}}, \\
\mathcal{M}_{i} & =\underset{j: y_{j} \neq y_{i}, j \neq i}{\operatorname{argmin}} \mathcal{D}_{\mathbf{v}_{i}, \mathbf{v}_{j}} .
\end{aligned}
$$

The margin of the $i$-th set is then defined as the difference of the distances between $\mathcal{M}_{i}$ and $\mathcal{H}_{i}$, as

$$
\rho_{i}=\mathcal{D}_{\mathbf{v}_{i}, \mathbf{v}_{\mathcal{M}_{i}}}-\mathcal{D}_{\mathbf{v}_{i}, \mathbf{v}_{\mathcal{H}_{i}}}
$$

where $\mathcal{H}_{i}^{y_{i}}$ is the nearest set from the same class of the $i$-th set, and $\mathcal{M}_{i}^{y_{i}}$ is the nearest one form a different class. The main difficulty here is that $\mathbf{v}_{i}$ is a variable to be solved when 
we compute the margin. Thus, it is impossible to directly find the nearest neighbors. To overcome this problem, following the principles of the expectation-maximization (EM) algorithm, we develop a probabilistic model where the nearest neighbors of a given set are treated as hidden variables. The probabilities of the $j$-th set being the nearest miss or hit of the $i$-th set is denoted as $P\left(j=\mathcal{M}_{i} \mid\left\{\mathbf{v}_{i}\right\}\right)$ and $P\left(j=\mathcal{H}_{i} \mid\left\{\mathbf{v}_{i}\right\}\right)$, respectively, and they are estimated via the standard kernel density estimation:

$$
\begin{gathered}
P\left(j=\mathcal{H}_{i} \mid\left\{\mathbf{v}_{i}\right\}\right)=\left\{\begin{array}{cc}
\frac{K_{\mathbf{v}_{i}, \mathbf{v}_{j}}}{\sum_{k: y_{k}=y_{i}} K_{\mathbf{v}_{i}, \mathbf{v}_{k}}}, & \text { if } y_{j}=y_{i}, \text { and } j \neq i \\
0, & \text { else }
\end{array}\right. \\
P\left(j=\mathcal{M}_{i} \mid\left\{\mathbf{v}_{i}\right\}\right)=\left\{\begin{array}{cc}
\frac{K_{\mathbf{v}_{i}, \mathbf{v}_{j}}}{\sum_{k: y_{k} \neq y_{i}} K_{\mathbf{v}_{i}, \mathbf{v}_{k}}} & \text { if } y_{j} \neq y_{i}, \text { and } j \neq i \\
0 & \text { else }
\end{array}\right.
\end{gathered}
$$

where $K_{\mathbf{v}_{i}, \mathbf{v}_{j}}=\exp \left(-\frac{\mathcal{D}_{\mathbf{v}_{i}, \mathbf{v}_{j}}}{2 \sigma^{2}}\right)$ is the Gaussian kernel function, and $\sigma$ is the band-width parameter. Subsequently the probabilistic margin is defined as

$$
\begin{aligned}
\rho_{i}= & \sum_{j=1}^{N} P\left(j=\mathcal{M}_{i} \mid\left\{\mathbf{v}_{i}\right\}\right) \times \mathcal{D}_{\mathbf{v}_{i}, \mathbf{v}_{j}} \\
& -\sum_{j=1}^{N} P\left(j=\mathcal{H}_{i} \mid\left\{\mathbf{v}_{i}\right\}\right) \times \mathcal{D}_{\mathbf{v}_{i}, \mathbf{v}_{j}}
\end{aligned}
$$

Apparently, the margin of each set should be maximized. Considering the representation error to be minimized and the margin to be maximized simultaneously, we have the following objective function regarding the parameters $\mathbf{v}_{i}$ and $\boldsymbol{\alpha}_{i}$ as follows

$$
\begin{aligned}
& \min _{\left\{\boldsymbol{\alpha}_{i}, \mathbf{v}_{i}\right\}} \sum_{i=1}^{N}\left\{\mathcal{R}_{\mathbf{v}_{i}, \boldsymbol{\alpha}_{i}}+\lambda\left\|\boldsymbol{\alpha}_{i}\right\|_{1}\right. \\
& +\gamma\left[\sum_{j=1}^{N} P\left(j=\mathcal{H}_{i} \mid\left\{\mathbf{v}_{i}\right\}\right) \times \mathcal{D}_{\mathbf{v}_{i}, \mathbf{v}_{j}}\right. \\
& \left.\left.-\sum_{j=1}^{N} P\left(j=\mathcal{M}_{i} \mid\left\{\mathbf{v}_{i}\right\}\right) \times \mathcal{D}_{\mathbf{v}_{i}, \mathbf{v}_{j}}\right]\right\} \text {, }
\end{aligned}
$$

where the $\left\|\boldsymbol{\alpha}_{i}\right\|_{1}$ is the $l_{1}$ norm based sparse term on $\boldsymbol{\alpha}_{i}$, and $\lambda$ and $\gamma$ are the trade-off weights, which are set by crossvalidation.

\section{B. Optimization}

We adopt the EM framework to optimize the objective function in (9) in an iterative algorithm. The algorithm is composed of two iterative steps: the E-step and the M-step.

1) E-step: In the E-step, we compute the probabilities of $P\left(j=\mathcal{H}_{i} \mid\left\{\mathbf{v}_{i}\right\}\right)$ and $P\left(j=\mathcal{M}_{i} \mid\left\{\mathbf{v}_{i}\right\}\right)$ based on $\left\{\mathbf{v}_{i}\right\}$ estimated in the previous iteration, as in (7).

2) M-Step: In the M-Step, we try to optimize (9) by fixing the probabilities. By denoting $P_{i j}=P\left(j=\mathcal{H}_{i} \mid\left\{\mathbf{v}_{i}\right\}\right)-$
$P\left(j=\mathcal{M}_{i} \mid\left\{\mathbf{v}_{i}\right\}\right)$, the objective function in (9) is reduced to

$$
\min _{\left\{\boldsymbol{\alpha}_{i}, \mathbf{v}_{i}\right\}} \sum_{i=1}^{N}\left(\mathcal{R}_{\mathbf{v}_{i}, \boldsymbol{\alpha}_{i}}+\lambda\left\|\boldsymbol{\alpha}_{i}\right\|_{1}+\gamma \sum_{j=1}^{N} P_{i j} \mathcal{D}_{\mathbf{v}_{i}, \mathbf{v}_{j}}\right)
$$

We optimize the representation parameters $\boldsymbol{\alpha}_{i}$ and $\mathbf{v}_{i}$ for each set one by one. To optimize $\boldsymbol{\alpha}_{i}$ and $\mathbf{v}_{i}$, we fix all the remaining representation parameters $\boldsymbol{\alpha}_{j}$ and $\mathbf{v}_{j}(j \neq i)$. Using the property $\mathcal{D}_{\mathbf{v}_{i}, \mathbf{v}_{i}}=0$ and $\mathcal{D}_{\mathbf{v}_{i}, \mathbf{v}_{j}}=\mathcal{D}_{\mathbf{v}_{j}, \mathbf{v}_{i}}$, we rewrite the optimization of objective of (10) with respect to only $\boldsymbol{\alpha}_{i}$ and $\mathbf{v}_{i}$ as follows:

$$
\min _{\boldsymbol{\alpha}_{i}, \mathbf{v}_{i}}\left\{\begin{array}{l}
H\left(\boldsymbol{\alpha}_{i}, \mathbf{v}_{i}\right) \\
=\mathcal{R}_{\mathbf{v}_{i}, \boldsymbol{\alpha}_{i}}+\lambda\left\|\boldsymbol{\alpha}_{i}\right\|_{1} \\
\quad+\gamma\left(\sum_{j: j \neq i} P_{i j} \mathcal{D}_{\mathbf{v}_{i}, \mathbf{v}_{j}}+\sum_{j: j \neq i} P_{j i} \mathcal{D}_{\mathbf{v}_{j}, \mathbf{v}_{i}}\right) \\
=\mathcal{R}_{\mathbf{v}_{i}, \boldsymbol{\alpha}_{i}}+\lambda\left\|\boldsymbol{\alpha}_{i}\right\|_{1}+\gamma \sum_{j: j \neq i}\left(P_{i j}+P_{j i}\right) \mathcal{D}_{\mathbf{v}_{i}, \mathbf{v}_{j}} \\
=f\left(\boldsymbol{\alpha}_{i}, \mathbf{v}_{i}\right)+g\left(\boldsymbol{\alpha}_{i}\right)
\end{array}\right\}
$$

The objective function $H\left(\boldsymbol{\alpha}_{i}, \mathbf{v}_{i}\right)$ in (11) is a composite model consisting of a smooth function $f\left(\boldsymbol{\alpha}_{i}, \mathbf{v}_{i}\right)=$ $\mathcal{R}_{\mathbf{v}_{i}, \boldsymbol{\alpha}_{i}}+\gamma \sum_{j: j \neq i}\left(P_{i j}+P_{j i}\right) \mathcal{D}_{\mathbf{v}_{i}, \mathbf{v}_{j}}$ and a non-smooth function $g\left(\alpha_{i}\right)=\lambda\left\|\boldsymbol{\alpha}_{i}\right\|_{1}$.

To solve the optimization problem in (11), we employ the accelerated proximal gradient (APG) algorithm introduced in [22]. In the $t$-th iteration, to obtain the new solution $\boldsymbol{\alpha}_{i}^{t}$ and $\mathbf{v}_{i}^{t}$, we solve the following proximal regularization problem based on the previous the solution $\boldsymbol{\alpha}_{i}^{t-1}$ and $\mathbf{v}_{i}^{t-1}$ :

$$
\boldsymbol{\alpha}_{i}^{t}, \mathbf{v}_{i}^{t}=\arg \min _{\boldsymbol{\alpha}_{i}, \mathbf{v}_{i}}\left\{\begin{array}{l}
Q_{L^{t}}\left(\boldsymbol{\alpha}_{i}, \boldsymbol{\beta}_{i}^{t}, \mathbf{v}_{i}, \mathbf{u}_{i}^{t}\right) \\
=\frac{L^{t}}{2}\left\|\boldsymbol{\alpha}_{i}-\left(\boldsymbol{\beta}_{i}^{t}-\frac{1}{L^{t}} \nabla f_{\boldsymbol{\beta}_{i}^{t}}\right)\right\|_{2}^{2} \\
+\frac{L^{t}}{2}\left\|\mathbf{v}_{i}-\left(\mathbf{u}_{i}^{t}-\frac{1}{L^{t}} \nabla f_{\mathbf{u}_{i}^{t}}\right)\right\|_{2}^{2} \\
+g\left(\boldsymbol{\alpha}_{i}\right)
\end{array}\right\},
$$

where

$$
\begin{aligned}
& \boldsymbol{\beta}_{i}^{t}=\boldsymbol{\alpha}_{i}^{t-1}+\frac{\tau^{t-1}-1}{\tau^{t}}\left(\boldsymbol{\alpha}_{i}^{t-1}-\boldsymbol{\alpha}_{i}^{t-2}\right), \\
& \mathbf{u}_{i}^{t}=\mathbf{v}_{i}^{t-1}+\frac{\tau^{t-1}-1}{\tau^{t}}\left(\mathbf{v}_{i}^{t-1}-\mathbf{v}_{i}^{t-2}\right),
\end{aligned}
$$

with $\tau^{t}=\frac{1+\sqrt{1+4 \tau^{t-1^{2}}}}{2}$ being the initial approximation of the next solution for $\boldsymbol{\alpha}_{i}^{t}, L^{t}=\eta^{i^{t}} L^{t-1}$ being the step size related to the Lipschitz constant, and $i^{t}$ being the smallest nonnegative integers such that $H\left(\boldsymbol{\alpha}_{i}^{t-1}, \mathbf{v}_{i}^{t-1}\right) \leq$ 


$$
\begin{aligned}
Q_{L^{t}}\left(\boldsymbol{\alpha}_{i}^{t-1}, \boldsymbol{\beta}_{i}^{t}, \mathbf{v}_{i}^{t-1}, \mathbf{u}_{i}^{t}\right), \text { and } \\
\nabla f_{\boldsymbol{\beta}_{i}^{t}}=\left.\frac{\partial f\left(\mathbf{v}_{i}, \boldsymbol{\alpha}_{i}\right)}{\partial \boldsymbol{\alpha}_{i}}\right|_{\boldsymbol{\alpha}_{i}=\boldsymbol{\beta}_{i}^{t}}=-2 X_{i}^{\top}\left(\mu_{i}+U_{i} v_{i}-X_{i} \boldsymbol{\beta}_{i}^{t}\right), \\
\nabla f_{\mathbf{u}_{i}^{t}}=\left.\frac{\partial f\left(\mathbf{v}_{i}, \boldsymbol{\alpha}_{i}\right)}{\partial \mathbf{v}_{i}}\right|_{\mathbf{v}_{i}=\mathbf{u}_{i}^{t}} \\
=2\left[1+\gamma \sum_{j: j \neq i}\left(P_{i j}+P_{j i}\right)\right] U_{i}^{\top}\left(U_{i} \mathbf{u}_{i}^{t}+\boldsymbol{\mu}_{i}\right) \\
-2 U_{i} X_{i} \boldsymbol{\alpha}_{i}-2 \gamma \sum_{j: j \neq i}\left(P_{i j}+P_{j i}\right)\left(\boldsymbol{\mu}_{j}-U_{j} \mathbf{u}_{j}^{t}\right) .
\end{aligned}
$$

It could be proved that when $g\left(\boldsymbol{\alpha}_{i}\right)$ is given as $g\left(\boldsymbol{\alpha}_{i}\right)=$ $\lambda\left\|\boldsymbol{\alpha}_{i}\right\|_{1}$, the optimal solution of (12) can be obtained by

$$
\begin{aligned}
\boldsymbol{\alpha}_{i}^{t} & =\tau_{\frac{\lambda}{L^{t}}}\left(\boldsymbol{\beta}_{i}^{t}-\frac{1}{L^{t}} \nabla f_{\boldsymbol{\beta}_{i}^{t}}\right), \\
\mathbf{v}_{i}^{t} & =\mathbf{u}_{i}^{t}-\frac{1}{L^{t}} \nabla f_{\mathbf{u}_{i}^{t}},
\end{aligned}
$$

where $\tau_{\varepsilon}(w)=(|w|-\varepsilon)_{+} \operatorname{sgn}(w)$ is the soft-thresholding operators. The final iterative representation algorithm for LaMa-IS method is summarized in Algorithm 1.

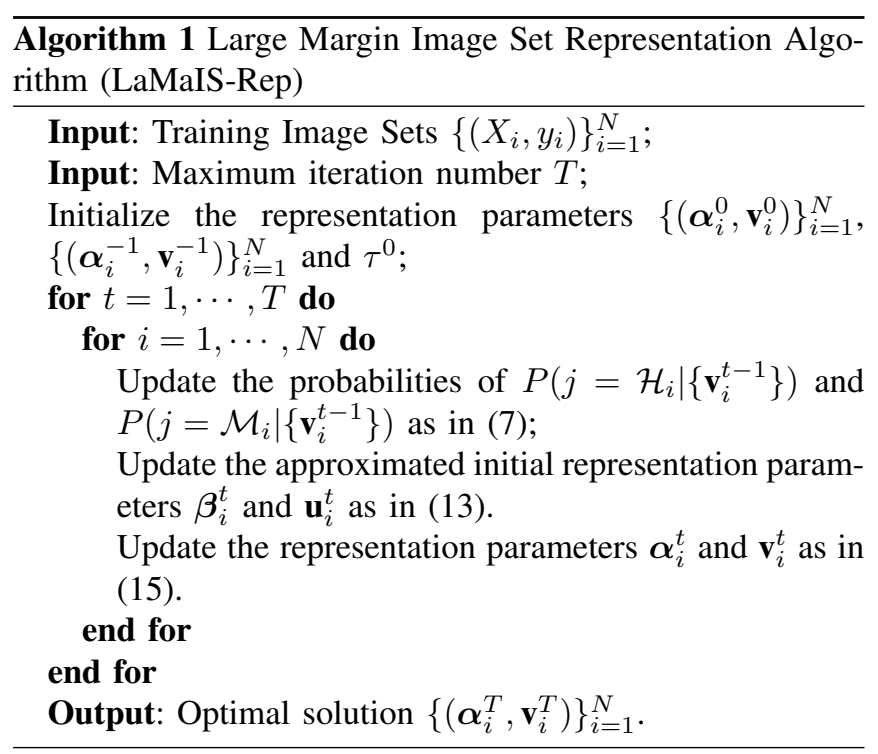

\section{Classification}

Given a test image set $X_{k}$, we first assume that it belongs to class $y \in\{1, \cdots, C\}$, and then compute the distance to the sets of class $y$ as

$$
\begin{aligned}
& \mathcal{E}_{y}=\min _{\boldsymbol{\alpha}_{k}, \mathbf{v}_{k}}\{ \mathcal{R}_{\boldsymbol{\alpha}_{k}, \mathbf{v}_{k}}+\lambda\left\|\boldsymbol{\alpha}_{k}\right\|_{1} \\
&+\gamma\left[\sum_{j=1}^{N} P\left(i=\mathcal{H}_{k} \mid\left\{\mathbf{v}_{i}\right\}, \mathbf{v}_{k}, y_{k}=y\right) \times \mathcal{D}_{\mathbf{v}_{k}, \mathbf{v}_{i}}\right. \\
& \\
&\left.\left.-\sum_{j=1}^{N} P\left(i=\mathcal{M}_{k} \mid\left\{\mathbf{v}_{i}\right\}, \mathbf{v}_{k}, y_{k}=y\right) \times \mathcal{D}_{\mathbf{v}_{k}, \mathbf{v}_{i}}\right]\right\},
\end{aligned}
$$

where $P\left(i=\mathcal{H}_{k} \mid\left\{\mathbf{v}_{i}\right\}, \mathbf{v}_{k}, y_{k}=y\right)$ is the probability of $X_{i}$ being the nearest hit of $X_{i}$ conditional on $y_{k}=y$. This problem can also be solved by an EM algorithm with the APG optimization. We then assign a label $y_{k}$ to $X_{k}$ as follows:

$$
y_{k}=\underset{y \in\{1, \cdots, C\}}{\operatorname{argmin}} \mathcal{E}_{y}
$$

\section{Time Complexity}

Given the iteration number $T$, in the off-line procedure of our method, the presentation parameters are updated one by one for $T$ times. Since there are $N$ training image sets, the training time complexity is $O(N \times T)$. In the online classification procedure, the test image set is compared to each class, and in each comparison, the representation parameter of the test image set is updated for $T$ times, and the representation parameters of the training image set are fixed. Since the number of classes is $C$, the time complexity for the on-line classification is $O(C \times T)$. In contrast, the SANP algorithm does not have a training procedure. The test image set is compared to all the training image sets. In each comparison, the parameter of both the test image and training image set are updated, thus the time complexity is $O(2 \times N \times T)$. Usually $C \ll N$, thus the time complexity is reduced significantly compared to SANP.

\section{EXPERIMENTS}

We evaluated the proposed method on two comprehensive face image set classification tasks where the goal is to conduct video-based face recognition.

\section{A. Dataset and Setup}

To evaluate our method, we used two real-world face video sequence datasets:

- Conference Face Set: We collected a human face video sequence dataset from an international academic conference of 5 days. The videos of 32 different conference participants' faces are captured during the oral session, poster session, reception banquet and some other casual scenes with an handheld camera. The face images extracted from the frames of this dataset cover large variations in illumination, head pose and facial expression, making the face recognition task from these videos quite challenging. For example, the oral presenters' faces were captured during both oral presentation procedure when light was off and Q\&A procedure when 
light was on. Moreover, the participants' expressions were quite serious when discussing academic problems, while much more relaxed during the reception banquet. All the images are taken from the same coordinates, but not always of the same sample size. The videos of 32 participants were first captured and then split into totally 507 continuous video sequences to construct the dataset. The number of video sequences for each participant varies from 12 to 19 . The entire dataset were further divided into a training set (328 sequences) and an independent test (179 sequences). The statistic information of the dataset is summarized in Table I. The frames of each sequence are considered as an image set, and the face region is firstly extracted, then the local binary pattern (LBP) features are extracted from the face region, and finally the LBP feature vectors are combined to construct the data matrix of an image set.

- YouTube Face Set: We also used a large-scale face image set database - YouTube Celebrities database [23]. It is the largest video database proposed for video face tracking and recognition. In this database, 1910 video sequences of 47 celebrities (actors, actresses and politicians) were collected from YouTube. The number of sequences for each celebrity varies from 17 to 108 . The number of frames of each sequences varies from 8 to 400, and most frames are of low resolution and highly compressed. This database is more challenging than other image set databases, due to the large variations in poses, illuminations and expressions. We also randomly split the database into a training set (1275 sequences) and an independent test set (635 sequences). The statistics of the training set and test set is given in Table II. The face area is cropped from each frame and scaled to size of $20 \times 20$, and the pixel values are used as the visual features after histogram equalization.

To conduct the experiments, we first performed the cross validation to the training sets to select the optimal parameters. The 8 -fold cross validation and 10 -fold cross validation were performed to the conference face database and the YouTube face database respectively. Using the parameters learned by the training set, we classified the image sets in the independent test sets. The classification accuracies are reported as the performance measure.

\section{B. Results}

We compared our method against the state-of-the-art image set classification methods, including DCC [23], MMD [5], MDA [15], AHISD [16], COV [19], and SANP [17], [18]. The performance of different methods on the conference face database is shown in Figure 1. In Figure 1 (a), the boxplots of the accuracies of the 8-fold cross validation is shown, while in Figure 1 (b), the accuracies of evaluation on the independent test set is given. It is obvious that LaMaIS outperforms all other methods on both the training and test sets. The better performance of LaMa-IS is mainly due to the usage of both the image set class labels and the exploration of the global structure of the image set database. MDA also matches the image samples against its neighbors of the same set and different sets, which is another strategy of the large margin framework. However, we defined the margin at the image set level, instead of the individual image sample level. Thus although MDA also archives good classification accuracy, LaMa-IS performs significantly better than MDA. SANP and LaMa-IS both use the same two models to represent the image sets, but SANP only focuses on the comparison of a pair of image sets, whereas LaMa-IS learns the representations for all the training image sets in a discriminant manner. It turns out that tuning the representation parameters coherently for all the image sets by using the class labels is not a trivial task, which can significantly improve the performance. This can be verified by the outperformance of LaMa-IS over SANP. COV represents each image set as a covariance matrix and also utilizes the class labels to learn the representation parameters. However, its performance is inferior to both LaMa-IS and SANP, which means that the representation model of using both image samples and affine hull model is more effective than the covariance matrix, especially when the training sample number is not large.

Figure 2 shows the performance of different methods on the YouTube face set. It can be seen that LaMa-IS also outperforms all other methods, on both the training set and test set. Different from the results in Figure 1, COV outperforms SANP slightly. The possible reason is that the YouTube database provides more image sets and thus provides sufficient discriminant information to be utilized by COV.

We further compared the computation time of different methods on the YouTube database for training and testing (classification of one image set), and reported the results in Figure 2 (c). It could be seen that the training procedure for MDA, MMD and DCC is quite time consuming, and at the same time, the accuracy of these three methods is not satisfactory. SANP, on the other hand, has quite high time cost on the on-line testing process, but requires no time on training as it does not require a training step. Its performance is also much better than MDA, MMD and DCC. Comparing with SANP, the proposed LaMa-IS method requires an off-line training procedure, which does not consume much time, but can boost the classification performance significantly on this large scale database. Additionally, its on-line classification procedure is also computationally efficient.

\section{CONCLUSION}

In this paper, we have proposed a novel large-margin-based image set representation and classification method, LaMaIS. To represent an image set, LaMa-IS encodes information from both the image samples in the set and their affine hull models. We defined the margin of an image set as the difference of the distance to its nearest image set from different classes and the distance to its nearest image set of the same class. The maximum margin is optimized by an expectation-maximization (EM) strategy with accelerated 
TABLE I

STATISTICS OF THE CONFERENCE FACE IMAGE SET DATABASE.

\begin{tabular}{|l|c|c|c|}
\hline Sets & Sample Number & Participant Number & Samples for Each Participant \\
\hline \hline Training Set & 328 & 32 & $8 \sim 13$ \\
Test Set & 179 & 32 & $3 \sim 7$ \\
\hline Entire Set & 507 & 32 & $12 \sim 19$ \\
\hline
\end{tabular}

TABLE II

Statistics of the Youtube face IMAgE SET Database.

\begin{tabular}{|l|c|c|c|}
\hline Sets & Sample Number & Person Number & Samples for Each Person \\
\hline \hline Training Set & 1910 & 47 & $11 \sim 72$ \\
Test Set & 635 & 47 & $6 \sim 26$ \\
\hline Entire Set & 1910 & 47 & $17 \sim 108$ \\
\hline
\end{tabular}

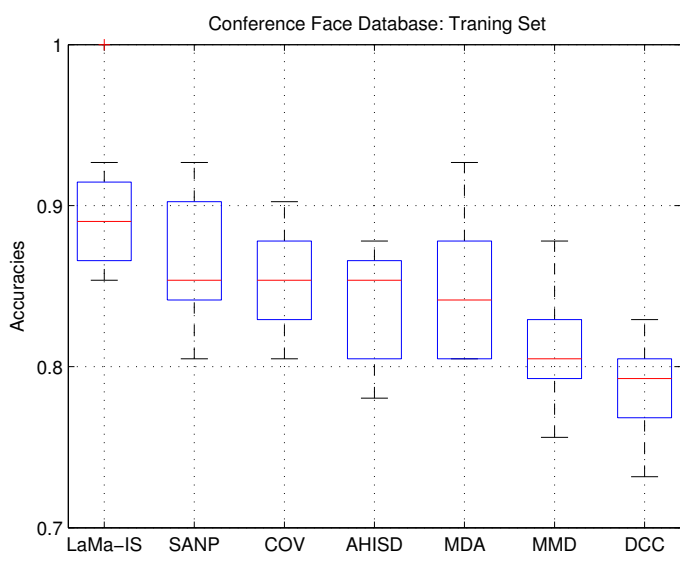

(a) 8-fold cross validation on the training set

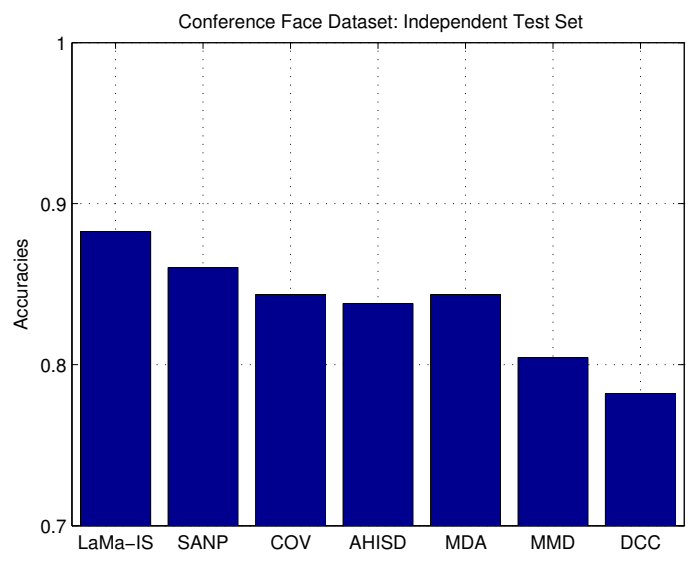

(b) Evaluation on the independent test set

Fig. 1. Performance of different methods on the conference face image set database.

proximal gradient (APG) optimization in an iterative algorithm. In the classification procedure, LaMa-IS compares a test image set to every class, instead of every image set, making it a computationally efficient algorithm for largescale applications. Experimental results on two comprehensive face image set classification tasks demonstrate that the proposed method significantly outperforms the state-of-theart methods in terms of both effectiveness and efficiency.

\section{REFERENCES}

[1] W. Zhao, R. Chellappa, P. Phillips, and A. Rosenfeld, "Face recognition: A literature survey," ACM Computing Surveys, vol. 35, no. 4, pp. 399-458, 2003.

[2] P. Jonathon Phillips, H. Moon, S. Rizvi, and P. Rauss, "The feret evaluation methodology for face-recognition algorithms," IEEE Transactions on Pattern Analysis and Machine Intelligence, vol. 22, no. 10, pp. 1090-1104, 2000.

[3] Y. Wang, W. Jiang, and G. Agrawal, "Scimate: A novel mapreducelike framework for multiple scientific data formats," in Cluster, Cloud and Grid Computing (CCGrid), 2012 12th IEEE/ACM International Symposium on. IEEE, 2012, pp. 443-450.

[4] J.-Y. Wang, I. Almasri, and X. Gao, "Adaptive graph regularized nonnegative matrix factorization via feature selection," in Pattern Recognition (ICPR), 2012 21st International Conference on. IEEE, 2012, pp. 963-966.
[5] R. Wang, S. Shan, X. Chen, and W. Gao, "Manifold-manifold distance with application to face recognition based on image set," in 26th IEEE Conference on Computer Vision and Pattern Recognition, CVPR, 2008.

[6] W.-S. Chu, J.-C. Chen, and J.-J. Lien, "Kernel discriminant transformation for image set-based face recognition," Pattern Recognition, vol. 44, no. 8, pp. 1567-1580, 2011.

[7] W. Fan and D.-Y. Yeung, "Face recognition with image sets using hierarchically extracted exemplars from appearance manifolds," in FGR 2006: Proceedings of the 7th International Conference on Automatic Face and Gesture Recognition, vol. 2006, 2006, pp. 177-182.

[8] T.-K. Kim, J. Kittler, and R. Cipolla, "Discriminative learning and recognition of image set classes using canonical correlations," IEEE Transactions on Pattern Analysis and Machine Intelligence, vol. 29, no. 6, pp. 1005-1018, 2007.

[9] T.-J. Chin, K. Schindler, and D. Suter, "Incremental kernel svd for face recognition with image sets," in FGR 2006: Proceedings of the 7th International Conference on Automatic Face and Gesture Recognition, vol. 2006, 2006, pp. 461-466.

[10] T. Wang and P. Shi, "Kernel grassmannian distances and discriminant analysis for face recognition from image sets," Pattern Recognition Letters, vol. 30, no. 13, pp. 1161-1165, 2009.

[11] O. Arandjelović, G. Shakhnarovich, J. Fisher, R. Cipolla, and T. Darrell, "Face recognition with image sets using manifold density divergence," in Proceedings of the IEEE Computer Society Conference on Computer Vision and Pattern Recognition, vol. 1, 2005, pp. 581-588.

[12] S. Wold, K. Esbensen, and P. Geladi, "Principal component analysis," Chemometrics and Intelligent Laboratory Systems, vol. 2, no. 1-3, pp. 


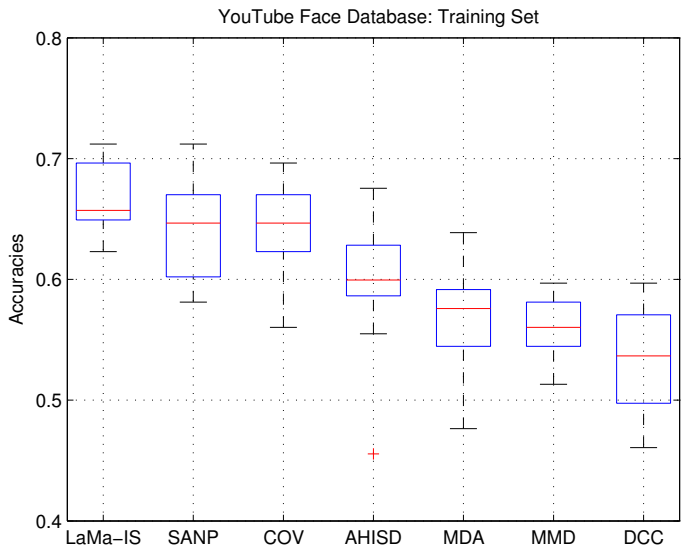

(a) 10-fold cross validation on the training set

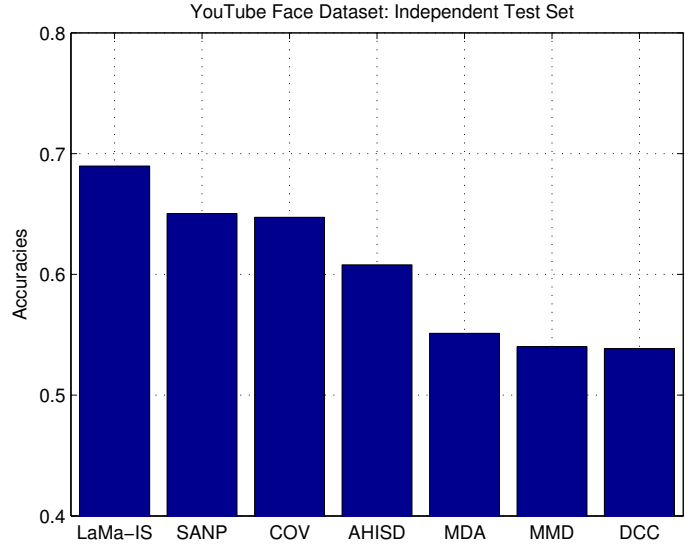

(b) Evaluation on the independent test set

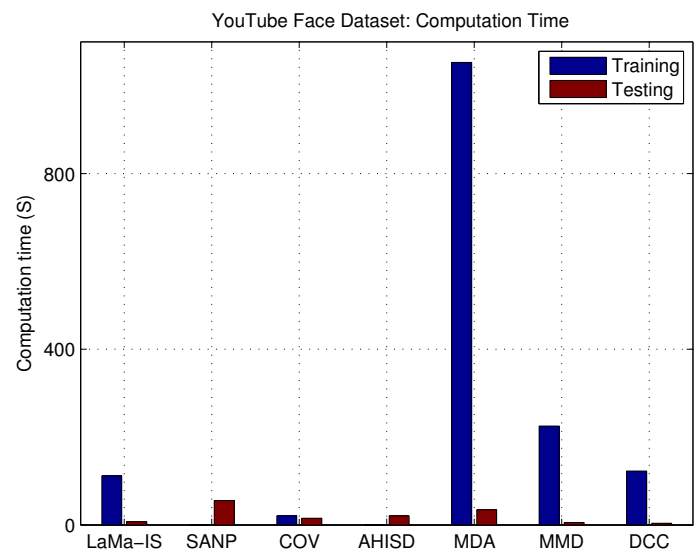

(c) Computation time

Fig. 2. Performance of different methods on the YouTube face image set database.

37-52, 1987.

[13] C. Cortes and V. Vapnik, "Support-vector networks," Machine Learning, vol. 20, no. 3, pp. 273-297, 1995.

[14] S. A. Dudani, "Distance-weighted k-nearest-neighbor rule." IEEE Transactions on Systems, Man and Cybernetics, vol. SMC-6, no. 4, pp. 325-327, 1976.

[15] R. Wang and X. Chen, "Manifold Discriminant Analysis," in CVPR: 2009 IEEE Conference on Computer Vision and Pattern Recognition, VOLS 1-4, 2009, pp. 429-436.

[16] H. Cevikalp and B. Triggs, "Face Recognition Based on Image Sets," in 2010 IEEE Conference on Computer Vision and Pattern Recognition (CVPR), 2010, pp. 2567-2573.

[17] Y. Hu, A. S. Mian, and R. Owens, "Sparse Approximated Nearest Points for Image Set Classification," in 2011 IEEE Conference on Computer Vision and Pattern Recognition (CVPR), 2011, pp. 121-128.

[18] — - "Face Recognition Using Sparse Approximated Nearest Points between Image Sets," IEEE Transactions on Pattern Analysis and Machine Intelligence, vol. 34, no. 10, pp. 1992-2004, 2012.

[19] R. Wang, H. Guo, L. S. Davis, and Q. Dai, "Covariance Discriminative Learning: A Natural and Efficient Approach to Image Set Classification," in 2012 IEEE Conference on Computer Vision and Pattern Recognition (CVPR), 2012, pp. 2496-2503.

[20] Y. Mu, W. Ding, and D. Tao, "Local discriminative distance metrics ensemble learning," Pattern Recognition, vol. 46, no. 8, pp. 2337$2349,2013$.

[21] T. Ahonen, A. Hadid, and M. Pietikinen, "Face description with local binary patterns: Application to face recognition," IEEE Transactions on Pattern Analysis and Machine Intelligence, vol. 28, no. 12, pp. 2037-2041, 2006.

[22] A. Beck and M. Teboulle, "A fast iterative shrinkage-thresholding algorithm for linear inverse problems," SIAM Journal on Imaging Sciences, vol. 2, no. 1, pp. 183-202, Mar. 2009.

[23] M. Kim, S. Kumar, V. Pavlovic, and H. Rowley, "Face tracking and recognition with visual constraints in real-world videos," in 26th IEEE Conference on Computer Vision and Pattern Recognition, CVPR, 2008. 